\title{
RESTITUCIÓN DE PTEROLEPIS GRALLATA (PANTEL, 1886) (ORTHOPTERA, TETTIGONIIDAE) COMO ESPECIE VÁLIDA Y ESTATUS TAXONÓMICO DE LAS ESPECIES IBÉRICAS DEL GÉNERO
}

\author{
P. Barranco ${ }^{1} \&$ J. Gutiérrez-Rodríguez ${ }^{2}$
}

\section{RESUMEN}

P. Barranco \& J. Gutiérrez-Rodríguez, 2010. Restitución de Pterolepis grallata (Pantel, 1886) como especie válida y estatus taxonómico de las especies ibéricas del género (Orthoptera, Tettigoniidae). Grael/sia, 66(2): 131-148.

Se revisa la situación taxonómica de las especies ibéricas encuadradas en los géneros Pterolepis Rambur, 1838 y Rhacocleis Fieber, 1853 mediante el estudio de 126 ejemplares procedentes de diversas instituciones y colecciones. Se restituye Pterolepis grallata (Pantel, 1886) como especie válida en función del estudio morfológico y filogenético. Se acepta el criterio de Willemse \& Willemse (2005), descartando la utilización de la espinación apical de las tibias posteriores como carácter para discriminar los géneros, por el que todas las especies ibéricas citadas hasta el momento en esos géneros, pertenecen al género Pterolepis.

Palabras clave: género Pterolepis; P. grallata; taxonomía; análisis filogenético; península Ibérica.

\begin{abstract}
P. Barranco \& J. Gutiérrez-Rodríguez, 2010. Restitution of Pterolepis grallata (Pantel, 1886) as a valid species and taxonomic status of the lberian species of the genus (Orthoptera, Tettigoniidae). Grael/sia, 66(2): 131-148 (in Spanish).

The taxonomic status of the Iberian species of the genera Pterolepis Rambur, 1838 and Rhacocleis Fieber, 1853 is revised through the study of 126 specimens from different private and institutional collections. Pterolepis grallata (Pantel, 1886) is reinstated as a valid species as a result of morphological studies and phylogenetic analysis. The criterion of Willemse \& Willemse (2005) not accepting the use of number of apical spurs of hind tibiae as a character for genera separation is followed, so that all Iberian species included up to now in these genera belong to genus Pterolepis.
\end{abstract}

Key words: genus Pterolepis; P. grallata; taxonomy; phylogenetic analysis; Iberian Peninsula.

Dpto. Biología Aplicada. Cite II-B. Universidad de Almería. 04120 Almería. España. pbvega@ual.es Museo Nacional de Ciencias Naturales. CSIC. C/ José Gutiérrez Abascal, 2. 28006 Madrid. España. jorge.gutierrez@mncn.csic.es 


\section{Introducción}

Las especies objeto de este estudio han estado encuadradas en los géneros Pterolepis Rambur, 1838, Rhacocleis Fieber, 1853 y Scirtobaenus Pantel, 1886. A lo largo de revisiones de los diferentes autores, se han sucedido las sinonimias y reubicación de las especies en un género u otro y se ha venido arrastrando un carácter inapropiado y erróneo para discriminar los dos géneros que aún se consideran.

Pantel (1886) describe el género Scirtobaenus a partir de material ibérico, indicando que es afín a los géneros Antaxius Brunner von Wattenwyl, 1882 y Rhacocleis, e incluye en él una única especie, $S$. grallatus Pantel, 1886.

Unos años después, Bolívar describe Scirtobaenus lusitanicus Bolívar, 1900 que diferencia de la anterior por la presencia de una espina en el margen superior interno de la tibia anterior y por la presencia de 3 espinas en el borde inferior del fémur anterior. En este mismo artículo describe Pterolepis cordubensis Bolívar, 1900 que se diferencia de $P$. spoliata Rambur, 1838 por las tegminas casi ocultas por el pronoto, aspecto más robusto, con las patas posteriores más cortas, décimo terguito del macho triangular agudo y cercos del mismo casi rectos, sólo un poco encorvados en el ápice.

Caudell (1908), en su obra sobre los Decticinae, establece unas claves para los géneros. Agrupándolos inicialmente en cinco grupos, de modo que los tres géneros anteriores quedaron ubicados en dos de estos grupos: grupo Rhacoclees (Scirtobaenus y Rhacocleis) y grupo Gampsoclees (Pterolepis). Estos grupos se separan por la presencia de 2 espinas apicales en el ápice de las tibias posteriores en el primer caso y 4 en el segundo. A continuación diferencia Scirtobaenus por el cerco del macho cónico con un diente en el borde interno y Rhacocleis con cerco del macho cónico. Añade además una llamada en la que indica que no conoce estos géneros, que la separación de ambos géneros la realiza siguiendo el criterio de otros autores y que el carácter utilizado parece inadecuado para este propósito. Conclusión que se estima acertada al contemplar la contradicción del carácter con la descripción y el dibujo de Pantel de $S$. grallatus (que él mismo recoge) y en el que se observa un diente basal interno en el cerco del macho. Por otro lado, Bolívar (1900) en su descripción de $S$. lusitanicus menciona que el cerco del macho es cónico, eludiendo cualquier alusión al diente interno en el mismo.
Uvarov (1934) establece la sinonimia entre Rhacocleis y Scirtobaenus indicando que después de estudiar los tipos de ambos géneros no puede establecer diferencias, por lo que reubica su especie S. turcicus Uvarov, 1930 como R. turcicus (Uvarov, 1930). A partir de entonces las dos especies ibéricas pasarían a denominarse Rhacocleis grallata (Pantel, 1886) y Rhacocleis lusitanica (Bolívar, 1900), tal y como recoge Harz (1969).

El género Pterolepis en la península Ibérica fue revisado y ampliado con la descripción de 6 nuevas especies por Galvagni (1981). Este autor partió de las 2 especies conocidas, una de ellas con dos subespecies: Pterolepis spoliata spoliata Rambur, 1838, P. s. minor Bolívar, 1900 y Pterolepis cordubensis Bolívar, 1900. Elevó a rango de especie la subespecie, P. minor Bolívar, 1900 y además describió $P$. nevadensis Galvagni, 1981, P. nadigi Galvagni, 1981, P. raggei Galvagni, 1981, P. llorenteae Galvagni, 1981, P. pascuali Galvagni, 1981 y P. kaltenbachi Galvagni, 1981. En 1985, Galvagni revisa las especies norteafrianas del género y confecciona una clave dicotómica para discriminar todas las especies, incluidas las ibéricas.

En 1988 K. G. Heller en su obra sobre los cantos de los tetigónidos europeos establece la sinonimia de casi todas las especies ibéricas de Pterolepis descritas por Galvagni con Pterolepis spoliata, incluyendo además la especie Rhacocleis grallata (Pantel, 1886), relegándolas al estatus de subespecie. Aduce para ello tanto aspectos morfológicos en los que no aprecia variaciones suficientes para garantizar el aislamiento reproductivo, como que el canto de todas las formas que estudió es coincidente. Compartimos que las especies ibéricas de Pterolepis descritas por Galvagni (1981) son muy difíciles de valorar y caracterizar, pues las diferencias en las estructuras señaladas por este autor para los machos son mínimas y pueden entenderse dentro de la variación intraespecífica y para las hembras no es posible. A nuestro criterio es necesaria la evaluación de las mismas mediante métodos genéticos para validar las identidades a nivel de especie o subespecie. Sin embargo discrepamos con que se haya incluido en ese grupo a $R$. grallata pues sus caracteres evidencian claramente que se trata de una especie válida diferente al resto de los Pterolepis ibéricos. Por último nos parece excesivamente aventurada y errónea, la propuesta de Heller con respecto a que las otras especies ibéricas de Pterolepis (P. cordubensis y R. lusitanica) podrí- 
an tratarse también de especies sinónimas de $P$. spoliata, pues existen suficientes diferencias en su morfología para que puedan ser consideradas como especies válidas.

Heller et al. (1998), en su listado de los ortópteros europeos indican que no existen caracteres que permitan separar los géneros Rhacocleis y Pterolepis, aludiendo a Heller (1988), y los consideran sinónimos, asignando todas las especies europeas al género Pterolepis. Pero en ese trabajo tampoco se realizó ninguna referencia taxonómica explícita en este sentido. Se puede decir que esta postura no se ha asumido posteriormente por parte de diversos autores que siguen considerando válidos los géneros Rhacocleis y Pterolepis (Otte, 1997; Ponel et al., 1998; Defaut, 2001; Willemse \& Willemse, 2005, 2008; Eades \& Otte, 2009).

En relación a las especies ibéricas, Otte (1997) en su listado mundial de tetigonoideos asume las sinonimias de Heller (1988) en cuanto a las especies ibéricas de Galvagni y su consideración como subespecies. Pero no en el caso de P. spoliata grallata, que considera como Rhacocleis spoliata grallata, dentro del género Rhacocleis, cometiendo el error de arrastrar la especie spoliata al género Rhacocleis, de modo que en su obra aparece esta especie en ambos géneros. Por un lado $P$. spoliata con 8 subespecies, (entre ellas $P$. spoliata grallata) y $R$. spoliata grallata, manteniendo también como válida $R$. lusitanica.

Heller et al. (1998) consideran que ambos géneros son sinónimos e incluyen a todas las especies europeas dentro de Pterolepis, incluida P. lusitani$c a$. Sin embargo Eades \& Otte (2009) en su listado digital asumen los criterios de Heller (1988), pero no los de Heller et al. (1998). De modo que en lo que concierne a las especies ibéricas, las especies de Galvagni junto con P. s. grallata son subespecies de $P$. spoliata, y Rhacocleis lusitanica sigue en su ubicación en el género como única especie ibérica del mismo.

Willemse \& Willemse (2005) abordan la situación de forma explícita, contemplando el estudio de 39 especies de ambos géneros (43 taxones incluyendo las subespecies). Sin embargo al final, concluyen sin adoptar ninguna solución taxonómica clara a nivel genérico, aunque rechazando la sinonimia postulada por Heller (1988). Relegan Pterolepis raggei Galvagni, 1981 a subespecie de Pterolepis spoliata, ya que esta especie fue omitida por Heller (1988), aunque ya estaba en Otte (1997).
Ponen en duda la asignación de varias especies de Rhacocleis proponiendo su asignación al género Pterolepis, si bien mantienen el género Rhacocleis con un buen número de especies, entre ellas Rhacocleis lusitanica. Además no se menciona, en ningún caso, ninguna combinación ni sinonimia nueva. Lo cual explica, como bien recogen estos mismos autores, que las especies ibéricas anteriormente integradas en Rhacocleis se encuentren en la actualidad asignadas en géneros diferentes.

En resumen, puede decirse que la situación de las especies ibéricas es caótica, por lo que en las siguientes páginas se intenta resolver utilizando caracteres morfológicos y moleculares.

\section{Material y Métodos}

\section{CARACTERES MORFOLÓGiCOS}

Para el estudio morfológico se han contemplado un total de 36 ejemplares de $P$. grallata y 79 de $P$. spoliata, el único ejemplar tipo conocido de $P$. cordubensis y 10 ejemplares de $P$. lusitanica. Todo ello de fauna ibérica y además se han estudiado 7 especímenes del género Pterolepis de especies europeas y 38 ejemplares de diferentes especies norteafricanas del género Rhacocleis. Todo este material está depositado en las colecciones que se enumeran a continuación. MNCN: colección del Museo Nacional de Ciencias Naturales de Madrid; PBV: colección Pablo Barranco Vega; AAS: colección Antonio Aguirre Segura; UAH: material cedido por la Universidad de Alcalá de Henares; UMU: material en préstamo de la Universidad de Murcia; UCM: material en préstamo de la Universidad Complutense de Madrid; UGR: material en préstamo del Dr. Felipe Pascual Torres, de la Universidad de Granada; CIBIO-UP: material en préstamo de Sónia Ferreira de Portugal y KGH: material en préstamo del Dr. Klaus-Gerhar Heller. La relación de los ejemplares estudiados se detalla en el Apéndice.

Todos los ejemplares se han estudiado morfológicamente mediante estereoscopio binocular y algunos de ellos se han diseccionado para la realización de fotografías mediante microscopía electrónica a presión variable.

\section{CARACTERES MOLECULARES}

Extracción, amplificación y secuenciación del ADN mitocondrial. 
Para los análisis filogéneticos se utilizaron cinco ejemplares de $P$. spoliata y tres de $P$. grallata (ver Tabla 5). También se analizó un ejemplar de Tessellana tessellata (Charpentier, 1825) para utilizarlo como grupo externo. Todas las muestras habían sido conservadas en etanol absoluto y mantenidas en congelador a $-20^{\circ} \mathrm{C}$.

Para la obtención de tejido muscular se seccionó el metatórax. Posteriormente en la extracción del ADN mitocondrial se utilizó el kit Charge Switch gDNA Micro Tissue (Invitrogen). Una vez extraído el ADN se almacenaron los extractos a una temperatura de $4^{\circ} \mathrm{C}$.

Para la amplificación por PCR de un fragmento del gen mitocondrial citocromo oxidasa I (cox1) se utilizaron los siguientes cebadores COI-H (Machordom et al., 2003) y LCO1490 (Folmer et al., 1994). El proceso de PCR consistió en una fase de desnaturalización de 5 min a $94^{\circ} \mathrm{C}$, seguido de 40 ciclos (desnaturalización, $94^{\circ} \mathrm{C}, 45 \mathrm{~s}$; apareamiento de los cebadores, $42^{\circ} \mathrm{C}, 45 \mathrm{~s}$; síntesis de $\mathrm{ADN}, 72^{\circ} \mathrm{C}, 1$ $\min$ ).

Las reacciones de PCR fueron desarrolladas en un volumen de $25 \mu 1$, conteniendo: $2 \mu 1$ de ADN, 1 unidad de Taq polimerasa (Biotools), 0,2 $\mu \mathrm{M}$ de cada uno de los cebadores, $0,4 \mathrm{mM}$ de dNTP, $1 \mathrm{mM}$ de $\mathrm{MgCl}_{2}$ y 2,5 $\mu$ l de tampón con $\mathrm{MgCl}_{2} 2 \mathrm{mM}$. Una vez amplificado el ADN se precipitaron los productos de PCR con acetato de sodio y etanol y fueron resuspendidos en $22 \mu \mathrm{l}$ de agua ultrapura. Las muestras se secuenciaron en un secuenciador automático ABI PRISM 3700. Las secuencias del fragmento de cox 1 se alinearon con PAUP*4.0b10 (Swofford, 2002).

\section{ANÁLISIS FILOGENÉTICO}

Se aplicaron dos métodos de análisis filogenéticos: máxima verosimilitud (ML) e inferencia bayesiana. Para identificar el modelo evolutivo al que mejor se ajustan las secuencias, se utilizó el programa Modeltest 3.7 (Posada \& Crandall, 1998). Los análisis de ML se realizaron con el programa PhyML 3.0 (Guindon \& Gascuel, 2003), analizando 500 réplicas de bootstrap para calcular la robustez de las ramas. El análisis bayesiano se llevó a cabo utilizando el programa Mr. Bayes 3.1.2 (Ronquist \& Huelsenbeck, 2003). Se realizaron dos ejecuciones de 1.000.000 de generaciones cada una, tomando muestras cada 1.000 generaciones. La convergencia de los análisis se testó con el programa Tracer 1.4 (Rambaut \& Drummond, 2007) y se eliminó el 10\% de las generaciones como "burnin". Se calcularon las divergencias genéticas medias (basadas en distancias sin corregir) entre las secuencias, mediante el programa PAUP*4.0b10 (Swofford, 2002).

\section{Resultados y Discusión}

La problemática que existe para caracterizar e identificar correctamente los géneros Pterolepis y Rhacocleis radica en que tal separación se basa en un carácter inconsistente y tremendamente variable dentro de la especie e incluso dentro de la misma población. Algunos autores incluso han manifestado la variación de este carácter en la serie típica sobre la que han descrito nuevas especies (Uvarov, 1942 y Galvagni, 1984).

Como exponen Willemse \& Willemse (2005), existe una gama de variaciones que se presentan en la forma de las espinas internas del ápice de la tibia posterior, que oscilan entre la forma típica de Pterolepis (con dos espinas bien formadas) a la forma típica de Rhacocleis (ausencia total de estructuras). Esta variación va desde las espinas bien constituidas, a reducción en el tamaño de las espinas (espinas cortas), pequeñas placas esclerotizadas, placa con una seta, minúscula placa o ausencia total de cualquier tipo de estructuras. Las "formas típicas" han sido hasta el momento el único carácter para discriminar entre ambos géneros, si bien como ya han constatado algunos autores, no son constantes y su variación sugiere la sinonimia de ambos géneros (Heller, 1988 y Heller et al., 1998) o la invalidez de este carácter (Uvarov, 1942; Willemse \& Willemse, 2005). Todos estos autores, postulan como carácter mucho más constante y válido la presencia de espinas en el margen dorsal interno de la tibia anterior. Así, las especies del género Pterolepis carecen de espinas y las del género Rhacocleis presentan de 1-3.

La clave de este artículo reside en el ejemplar que Heller identificó como $P$. grallata y que amablemente nos ha cedido para su estudio. Si bien existen dos problemas al abordar esta situación: uno referente a la especie y otro referente al género. En cuanto a la especie, la confusión reside en el error de la determinación del ejemplar mencionado.

En este estudio se evidencia que la presencia o ausencia de espinas internas en el ápice de las tibias posteriores y su grado de desarrollo o tipología, es un 
Tabla 1.- Espinulación de Pterolepis grallata. Tibia anterior: número de espinas en el siguiente orden: DE dorsales externas, DI dorsales internas, VE ventrales externas y VI ventrales internas. Tibia posterior: número de espinas apicales centrales en el ápice de las tibias derecha e izquierda: ${ }^{(*)}$ espina corta, 0 estructura escamiforme. Los valores son siempre pares. Para valores 1 , se entiende una espina y un proceso escamiforme, para valores 0 , se entienden dos procesos escamiformes. Cuando sólo aparece un valor, indica que el ejemplar carece de esa pata.

Table 1.- Leg spurs in Pterolepis grallata. Fore tibia: number of spurs in the following order: DE upper outer margin, DI upper iner margin, VE outer lower margin and VI inner lower margin. Hind tibia: number of mid spurs in the tip of right and left legs: $\left.{ }^{*}\right)$ short spur, 0 rudimentary sockets. The values are always pair. The value 1 means one spur and one rudimentary socket, value 0 means two rudimentary sockets. When just one value is given, it means that one leg is missing.

\begin{tabular}{|c|c|c|c|c|c|c|}
\hline \multicolumn{7}{|c|}{ Pterolepis grallata } \\
\hline \multirow[t]{2}{*}{ Localidad } & \multirow[t]{2}{*}{ Sexo } & \multicolumn{4}{|c|}{ Tibia anterior } & \multirow{2}{*}{$\begin{array}{c}\text { Tibia posterior } \\
\text { derecha-izquierda }\end{array}$} \\
\hline & & $\mathrm{DE}$ & DI & VE & VI & \\
\hline Uclés. Cotipo. MNCN & macho & 3 & 0 & 6 & 6 & $0-0$ \\
\hline Uclés. Cotipo. MNCN & hembra & -- & -- & -- & -- & $0-$ \\
\hline Cast. Branc. MNCN & macho & 4 & 0 & 6 & 6 & $0-$ \\
\hline Cast. Branc. MNCN & macho & 4 & 0 & 6 & 6 & $0-0$ \\
\hline Jándula. MNCN & macho & $4 / 3$ & 0 & 6 & 6 & $0-0$ \\
\hline Jándula. MNCN & hembra & 3 & 0 & 6 & 6 & $1^{*}-1^{*}$ \\
\hline Talavera. MNCN & hembra & 3 & 0 & 6 & 6 & $0-1^{\star}$ \\
\hline Brunete. MNCN & macho & 4 & 0 & 6 & 6 & $0-0$ \\
\hline Brunete. MNCN & macho & 4 & 0 & 6 & 6 & $0-0$ \\
\hline Córdoba. MNCN & hembra & 4 & 0 & 6 & 6 & $0-0$ \\
\hline El Pardo. MNCN & macho & 4 & 0 & 6 & 6 & $0-0$ \\
\hline Ponte do Sabor. PBV & hembra & 4 & 0 & 6 & 6 & $0-0$ \\
\hline Córdoba. PBV & macho & 4 & 0 & 6 & 6 & $-1^{\star}$ \\
\hline Tiesas Quintanal. PBV & macho & 4 & 0 & 6 & 6 & $1^{*}-2^{*}$ \\
\hline El Porrosillo. PBV & macho & 4 & 0 & 6 & 6 & $1-0$ \\
\hline El Porrosillo. PBV & macho & 4 & 0 & 6 & 6 & $1^{*}-2^{*}$ \\
\hline Villamanta. UAH & macho & 4 & 0 & 6 & 6 & $1^{*}-0$ \\
\hline Villa del Prado. UAH & ninfa & 4 & 0 & 6 & 6 & $0-0$ \\
\hline Orusco. UAH & ninfa & $3 / 4$ & 0 & 6 & 6 & $0-0$ \\
\hline Orusco. UAH & ninfa & 4 & 0 & 6 & 6 & $0-0$ \\
\hline Villa del Prado. UAH (SEM) & macho & $3 / 4$ & 0 & 6 & 6 & $0-0$ \\
\hline Villamanta. UAH & macho & 3 & 0 & 6 & 6 & $0-0$ \\
\hline S. Martín Vega. UAH & macho & 3 & 0 & 6 & 6 & $0-0$ \\
\hline Villaviciosa Odón. UAH & hembra & 3 & 0 & 6 & 6 & $0-0^{*}$ \\
\hline Villa del Prado. UAH (SEM) & hembra & 4 & 0 & 6 & 6 & $1^{*}-2^{*}$ \\
\hline Orusco. UAH & hembra & 4 & 0 & 6 & 6 & $0-1^{\star}$ \\
\hline Chapinería. UAH & hembra & 4 & 0 & 6 & 6 & $0-0$ \\
\hline Villamanta. UAH & macho & $3 / 4$ & 0 & 6 & 6 & $-1^{*}$ \\
\hline Chapinería. UAH & hembra & 3 & 0 & 6 & 6 & $0-0$ \\
\hline Orusco. UAH & hembra & 3 & 0 & 6 & 6 & $0-0$ \\
\hline Amieiro. CIBIO-UP & macho & 4 & 0 & 6 & 6 & $1^{*}-1^{*}$ \\
\hline Palão. CIBIO-UP & macho & 4 & 0 & 6 & 6 & $0-0$ \\
\hline Monte Barata. CIBIO-UP & hembra & 4 & 0 & 6 & 6 & $0-0$ \\
\hline
\end{tabular}

carácter polimórfico y variable incluso dentro de la misma especie y población, e incluso entre las patas derecha e izquierda de un mismo individuo para las especies ibéricas (Tablas 1-4). Esta circunstancia ya fue señalada por Willemse \& Willemse (2005). Por otro lado, en las Tablas 1-4 también se recoge la disposición de las espinas internas dorsales de la tibia anterior de todo el material estudiado. Salvo en dos 
Tabla 2.- Espinulación de Pterolepis spoliata. Tibia anterior: número de espinas en el siguiente orden: DE dorsales externas, DI dorsales internas, VE ventrales externas y VI ventrales internas. Tibia posterior: número de espinas apicales internas en el ápice de las tibias derecha e izquierda: (*) espina corta, 0 estructura escamiforme, $0^{+}$estructura impar. Los valores son siempre pares. Para valores 1 , se entiende una espina y un proceso escamiforme, para valores 0 , se entienden dos procesos escamiformes. Cuando sólo aparece un valor, indica que el ejemplar carece de esa pata.

Table 2.- Leg spurs in Pterolepis spoliata. Fore tibia: number of spurs in the following order: DE upper outer margin, DI upper iner margin, VE outer lower margin and $\mathrm{VI}$ inner lower margin. Hind tibia: number of mid spurs in the tip of right and left legs: ${ }^{(*)}$ short spur, 0 rudimentary sockets, $0^{+}$unpaired rudimentary socket. The values are always pair. The value 1 means one spur and one rudimentary socket, value 0 means two rudimentary sockets. When just one value is expresed, it means that one leg is missing.

\begin{tabular}{|c|c|c|c|c|c|c|}
\hline \multicolumn{7}{|c|}{ Pterolepis spoliata } \\
\hline \multirow[t]{2}{*}{ Localidad } & \multirow[t]{2}{*}{ Sexo } & \multicolumn{4}{|c|}{ Tibia anterior } & \multirow{2}{*}{$\begin{array}{c}\text { Tibia posterior } \\
\text { derecha-izquierda }\end{array}$} \\
\hline & & DE & DI & VE & VI & \\
\hline Alicante. $\mathrm{KGH}$ & macho & $4 / 5$ & 0 & 6 & 6 & $0-0$ \\
\hline Algeciras. MNCN & macho & 4 & 0 & 6 & 6 & $2-2$ \\
\hline Chiclana. Cat. Tipos 266. MNCN & macho & 4 & 0 & 6 & 6 & $1^{*}-1^{*}$ \\
\hline Chiclana. Cat. Tipos 267. MNCN & macho & 4 & 0 & 6 & 6 & $1-1$ \\
\hline Chiclana. Cat. Tipos 268. MNCN & hembra & 4 & 0 & 6 & 6 & $2-1^{*}$ \\
\hline Chiclana. Cat. Tipos 269. MNCN & hembra & 4 & 0 & 6 & 6 & $2^{*}-2^{*}$ \\
\hline Doñana. Cat. Tipos 7320. MNCN & macho & 4 & 0 & 6 & 6 & $2^{*}-2^{*}$ \\
\hline Paterna. MNCN & hembra & 4 & 0 & 6 & 6 & $2-2$ \\
\hline Los Montes. Málaga. MNCN & macho & 4 & 0 & 6 & 6 & $0^{+}-2$ \\
\hline Cartagena. MNCN & hembra & 4 & 0 & 7 & 7 & $0-1$ \\
\hline Cehegin. Murcia. MNCN & hembra & $3 / 4$ & 0 & 6 & 6 & $1 *-0$ \\
\hline Fondón. Almería. MNCN & hembra & 4 & 0 & 6 & 6 & $0-$ \\
\hline Málaga. MNCN & macho & 4 & 1 & 6 & 6 & $2-2$ \\
\hline Algeciras. MNCN & macho & 4 & 0 & 6 & 6 & $2-$ \\
\hline Cartagena. MNCN & macho & 4 & 0 & 6 & 6 & $2-2$ \\
\hline $\mathrm{S}^{\mathrm{a}}$ Carbón. Algeciras. MNCN & hembra & 4 & 0 & 6 & 6 & $2-2$ \\
\hline (sin localidad) MNCN & hembra & 4 & 0 & 6 & 6 & $2-2$ \\
\hline Granada. MNCN & hembra & $4 / 3$ & 0 & 6 & 6 & $0-0$ \\
\hline Cartagena. MNCN & hembra & $4 / 5$ & 0 & 6 & 6 & $1-2$ \\
\hline Jándula. MNCN & hembra & $4 / 3$ & 0 & 6 & 6 & -2 \\
\hline Algeciras. KGH & macho & 4 & 0 & 6 & 6 & $2-2$ \\
\hline Algeciras. KGH & hembra & 4 & 0 & 6 & 6 & $2-2$ \\
\hline Granada. KGH & macho & 4 & 0 & 6 & 6 & $2 *-2$ \\
\hline Granada. KGH & hembra & 4 & 0 & 6 & 6 & $0-0$ \\
\hline Málaga. KGH & hembra & $4 / 3$ & 0 & 6 & 6 & $2-2$ \\
\hline S A Ayllo. Jaén. PBV & macho & 4 & 0 & $5 / 6$ & $5 / 6$ & $0-0$ \\
\hline Albóx. Almería. PBV & macho & 4 & 0 & 6 & 6 & $1-1$ \\
\hline Taberno. Almería. PBV & macho & 4 & 0 & 6 & 6 & $2-2$ \\
\hline Taberno. Almería. PBV & macho & 4 & 0 & 6 & 6 & $2-2$ \\
\hline Taberno. Almería. PBV & macho & 4 & 0 & 6 & 6 & $0-0$ \\
\hline Sa Gádor. Alhama. PBV & hembra & 4 & 0 & 6 & 6 & $0-0$ \\
\hline $\mathrm{S}^{\mathrm{a}}$ Cabrera. Almería. PBV & hembra & 4 & 0 & 6 & 6 & $1-0$ \\
\hline Laujar. Almería. PBV & hembra & 4 & 0 & 6 & 6 & $1-1$ \\
\hline Fondón. Almería. PBV & hembra & 4 & 0 & 6 & 6 & $2-0$ \\
\hline Almuñecar. Granada. PBV & hembra & 4 & 0 & 6 & 6 & $2-2$ \\
\hline Sorbas. Almería. PBV & hembra & 3 & 0 & 6 & 6 & $1-1$ \\
\hline Sa Gádor. Enix. PBV & hembra & 4 & 0 & 6 & 6 & $1-1$ \\
\hline Antequera. Málaga. PBV & macho & 4 & 0 & 6 & 6 & $2-2$ \\
\hline Los Villares. Córdoba. PBV & macho & 4 & 0 & 6 & 6 & $2-2$ \\
\hline Los Villares. Córdoba. PBV & hembra & 4 & 0 & 6 & 6 & $2-2$ \\
\hline Montoro. Córdoba. PBV & macho & 4 & 0 & 6 & 6 & $2-$ \\
\hline S. M. Trassierra. Córdoba. PBV & macho & 4 & 0 & 6 & 6 & $2-2$ \\
\hline
\end{tabular}


Tabla 2.- Continuación.

Table 2.- Continued.

\begin{tabular}{|c|c|c|c|c|c|c|}
\hline \multicolumn{7}{|c|}{ Pterolepis spoliata } \\
\hline \multirow[t]{2}{*}{ Localidad } & \multirow[t]{2}{*}{ Sexo } & \multicolumn{4}{|c|}{ Tibia anterior } & \multirow{2}{*}{$\begin{array}{c}\text { Tibia posterior } \\
\text { derecha-izquierda }\end{array}$} \\
\hline & & $\mathrm{DE}$ & DI & VE & VI & \\
\hline S. Lúcar. Cádiz. PBV & hembra & 4 & 0 & 6 & 6 & $1-2$ \\
\hline Aguilas. Murcia. UMU & macho & 4 & 0 & 6 & 6 & $0-0$ \\
\hline Lorca. Murcia. UMU & macho & $3 / 4$ & 0 & 6 & 6 & $1-$ \\
\hline Lorca. Murcia. UMU & hembra & 4 & 0 & 6 & 6 & $0-0$ \\
\hline Lorca. Murcia. UMU & hembra & 4 & 0 & 6 & 6 & $1-2$ \\
\hline Colmenar Viejo. Madrid. UCM & hembra & 4 & 0 & 6 & 6 & $2-2$ \\
\hline Aravaca. Madrid. UCM & hembra & 4 & 0 & 6 & 6 & $2-2$ \\
\hline Cabo de Gata. Almería. AAS & hembra & 4 & 0 & 6 & 6 & $0^{+}-1$ \\
\hline Cabo de Gata. Almería. AAS & hembra & $3 / 4$ & 0 & 6 & 6 & $0-0$ \\
\hline Cabo de Gata. Almería. AAS & hembra & $3 / 4$ & 0 & 6 & 6 & $1-0$ \\
\hline Cabo de Gata. Almería. AAS & macho & $3 / 4$ & 0 & 6 & 6 & $2-1$ \\
\hline Cabo de Gata. Almería. AAS & macho & 4 & 0 & 6 & 6 & $0-0$ \\
\hline Cabo de Gata. Almería. AAS & macho & 4 & 0 & 6 & 6 & $0-0$ \\
\hline Cabo de Gata. Almería. AAS & macho & 4 & 0 & 6 & 6 & $1-1$ \\
\hline Cabo de Gata. Almería. AAS & macho & 4 & 0 & 6 & 6 & $1-0$ \\
\hline Cabo de Gata. Almería. AAS & macho & 4 & 0 & 6 & 6 & $0-0$ \\
\hline Cabo de Gata. Almería. AAS & hembra & 4 & 0 & 6 & 6 & $2-1$ \\
\hline Cabo de Gata. Almería. AAS & macho & 4 & 0 & 6 & 6 & -1 \\
\hline Cabo de Gata. Almería. AAS & macho & 4 & 0 & 6 & 6 & $2-0$ \\
\hline Cabo de Gata. Almería. AAS & macho & 4 & 0 & 6 & 6 & -0 \\
\hline Cabo de Gata. Almería. AAS & macho & 4 & 0 & 6 & 6 & $0-0$ \\
\hline Cabo de Gata. Alm. AAS (SEM) & macho & 4 & 0 & 6 & 6 & $1-1$ \\
\hline Cabo de Gata. Alm. AAS (SEM) & hembra & 4 & 0 & 6 & 6 & $1-2$ \\
\hline Lancha Cenes. Granada. UGR & hembra & $3 / 4$ & 0 & 6 & 6 & $2-0$ \\
\hline Huetor-Santillán. Granada. UGR & hembra & 4 & 0 & 6 & 6 & $2-2$ \\
\hline Valle Lanjarón. Granada. UGR & macho & $4 / 5$ & 0 & 6 & 6 & $2-2$ \\
\hline Valle Lanjarón. Granada. UGR & hembra & $3 / 4$ & 0 & 6 & 6 & $1-$ \\
\hline
\end{tabular}

individuos, que se pueden considerar anómalos pues además son asimétricos, de los 118 ejemplares ibéricos utilizados en el estudio morfológico, 116 carecen de espinas en el borde superior interno de la tibia anterior. En consonancia con este razonamiento, todas las especies ibéricas incluidas previamente en los géneros Rhacocleis, Scirtobaenus y Pterolepis se encuadrarían, según el criterio de Willemse \& Willemse (2005) confirmado por nosotros, en el género Pterolepis.

En la Tabla 6 se expone los caracteres morfológicos que identifican cada una de las especies ibéricas del género Pterolepis para ambos sexos.

\section{ANÁLISIS FILOGENÉTICO}

Un total de $658 \mathrm{pb}$ del gen cox 1 fueron alineadas para 8 ejemplares del género Pterolepis. No se detectaron inserciones ni deleciones. Las secuencias de cox 1 de Pterolepis poseen información filogenética en 97 (14,74\% del total) de las 104 posiciones que son variables. Las frecuencias de los nucleótidos son: $27,71 \%$ adenina, $21,33 \%$ citosina, $16,88 \%$ guanina y $34,06 \%$ timina. De acuerdo al criterio de información de Akaike (AIC), el modelo evolutivo al que mejor se ajustan las secuencias de Pterolepis fue el GTR + I.

Mediante los análisis de ML e inferencia bayesiana se obtuvieron dos árboles muy similares (Fig. 2). En los dos filogramas, las secuencias de Pterolepis se agrupan en dos clados principales, A y B. Ambos clados presentan altos valores de bootstrap y probabilidad posterior. El clado A agrupa los individuos asignados morfológicamente a Pterolepis spoliata y el clado B los de Pterolepis grallata. 

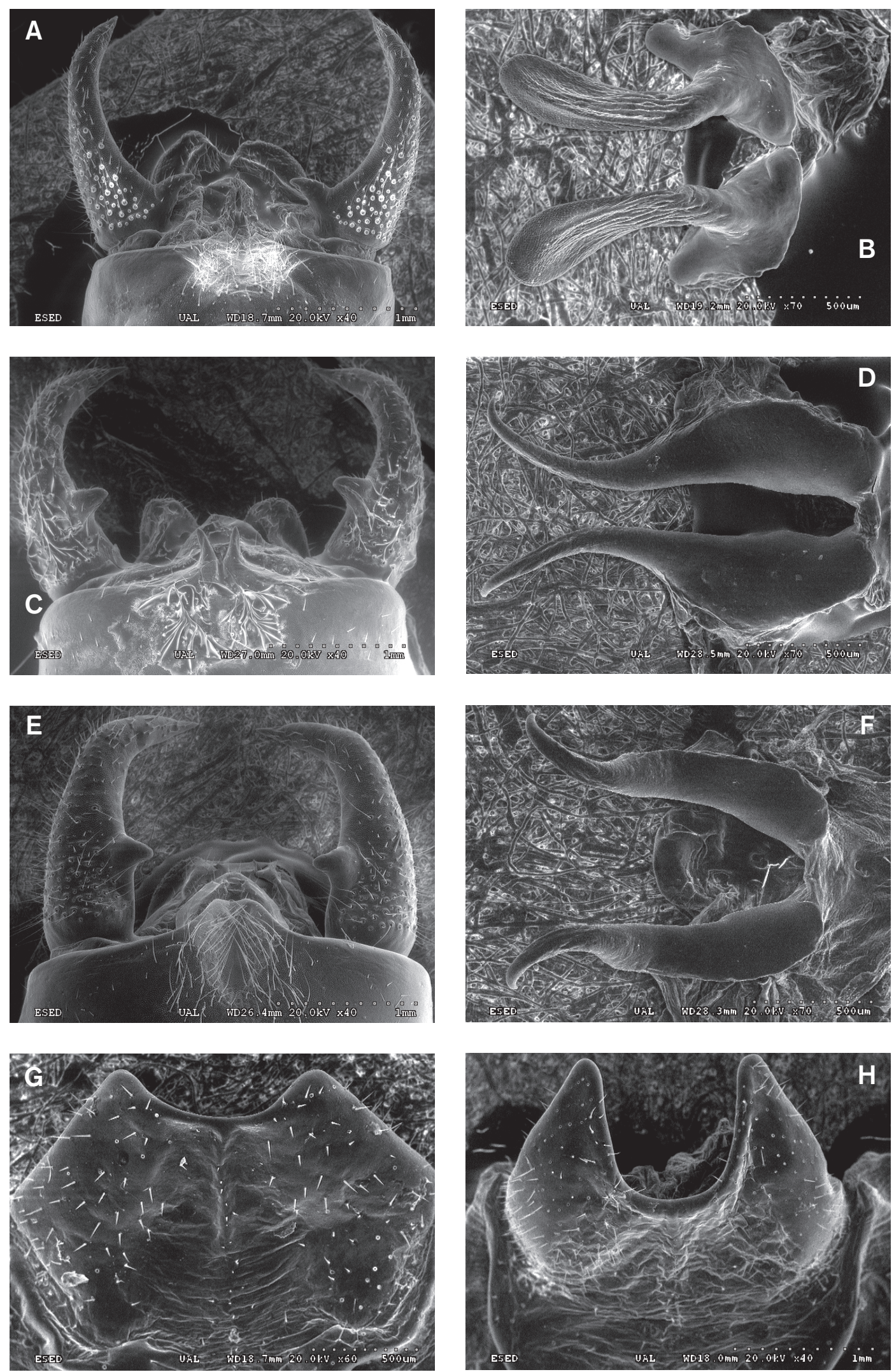

Graellsia, 66(2), Diciembre 2010, pp. 131-148 - ISSN: 0367-5041 doi:10.3989/graellsia.2010.v66.008 
Tabla 3.- Espinulación de Pterolepis lusitanica. Tibia anterior: número de espinas en el siguiente orden: DE dorsales externas, DI dorsales internas, VE ventrales externas y VI ventrales internas. Tibia posterior: número de espinas apicales internas en el ápice de las tibias derecha e izquierda: ${ }^{(*)}$ espina corta, 0 estructura escamiforme. Los valores son siempre pares: para valores 1, se entiende una espina y un proceso escamiforme, para valores 0 , se entienden dos procesos escamiformes. Cuando sólo aparece un valor, indica que el ejemplar carece de esa pata.

Tabla 3.- Leg spurs in Pterolepis lusitanica. Fore tibia: number of spurs in the following order: DE upper outer margin, DI upper iner margin, VE outer lower margin and VI inner lower margin. Hind tibia: number of mid spurs in the tip of right and left legs: ${ }^{(*)}$ short spur, 0 rudimentary sockets. The values are always pair. The value 1 means one spur and one rudimentary socket, value 0 means two rudimentary sockets. When just one value is expresed, it means that one leg is missing.

\begin{tabular}{llccccc}
\hline & \multicolumn{7}{c}{ Pterolepis lusitanica } \\
\hline Localidad & sexo & \multicolumn{3}{c}{ Tibia anterior } & Tibia posterior \\
& & DE & DI & VE & VI & derecha-izquierda \\
\hline Cast. Branco, Lectotipo. MNCN & macho & 4 & $1 / 0$ & $5 / 6$ & 6 & $2-2$ \\
MNCN & hembra & 4 & 0 & -- & -- & $0-0$ \\
MNCN & macho & 4 & 0 & 6 & 6 & $2-2$ \\
Monte Barata. CIBIO-UP (SEM) & macho & 4 & 0 & 6 & 6 & $2^{*}-2^{*}$ \\
Monte Barata. CIBIO-UP & macho & 4 & 0 & 6 & 6 & $2^{*}-2^{*}$ \\
Monchique. CIBIO-UP & macho & 4 & 0 & 6 & 6 & $0-0$ \\
Odemira. CIBIO-UP & macho & 4 & 0 & 6 & 6 & $2-2$ \\
Odemira. CIBIO-UP & macho & 4 & 0 & 6 & 6 & $1-2$ \\
Silves. CIBIO-UP & macho & 4 & 0 & 6 & 6 & $0-1$ \\
Silves. CIBIO-UP & hembra & 4 & 0 & 6 & 6 & $1^{*}-0$ \\
\hline
\end{tabular}

La divergencia genética entre las dos especies oscila entre $12,15 \%$ y $13,22 \%$ (ver Tabla 7 ). Estos valores de divergencia son altos entre especies del mismo género, si se compara con la divergencia interespecífica entre las especies del género Platycleis de la península Ibérica (5,07-11,70\%, datos sin publicar). Estos datos, junto con los caracteres morfológicos, apoyan la restitución de $P$. grallata como especie valida.

A su vez, el clado A está dividido en dos subclados (A1 y A2). El subclado A1 contiene los individuos de P. spoliata de Granada y el subclado A2 los de Sevilla. Los dos subclados están bien diferenciados. La divergencia genética entre las dos poblaciones de Pterolepis spoliata varía de 5,02 a 5,50 (ver Tabla 7). Estos valores sugieren una necesaria revisión de este taxón, ya que por ejemplo los nive- les de divergencia son similares a los encontrados entre Platycleis intermedia intermedia (Serville, 1839) y Platycleis albopunctata albopunctata (Goeze, 1778) (datos sin publicar).

\section{Pterolepis grallata (Pantel, 1886) stat. rest.}

Scirtobaenus grallatus Pantel, 1886: 253 - Caudell, 1908: 5

Rhacocleis grallata (Pantel): Harz, 1969: 440

Pterolepis spoliata grallata (Pantel): Heller, 1988: 143 Willemse \& Willemse, 2005: 263-269

Rhacocleis spoliata grallata (Pantel): Otte, 1997: 295

\section{Bona species, no sinonimia de Pterolepis spoliata}

Una vez estudiados los 33 ejemplares de esta especie, incluidos los tipos depositados en el MNCN de Madrid y el ejemplar de K. G. Heller

Fig. 1.- Pterolepis grallata. A: cercos y $10^{\circ}$ terguito del macho, B: titiladores, G: placa subgenital de la hembra. Pterolepis spoliata. C: cercos y $10^{\circ}$ terguito del macho, D: titiladores, $\mathrm{H}$ : placa subgenital de la hembra. Pterolepis lusitanica. E: cer$\cos$ y $10^{\circ}$ terguito del macho, F: titiladores.

Fig. 1.- Pterolepis grallata. A: male cerci and 10th abdominal tergite, B: titillators, G: female subgenital plate. Pterolepis spoliata. C: male cerci and 10th abdominal tergite, D: titillators, $\mathrm{H}$ : female subgenital plate. Pterolepis lusitanica. E: male cerci and 10th abdominal tergite, F: titillators. 
Tabla 4.- Espinulación de Pterolepis cordubensis. Tibia anterior: número de espinas en el siguiente orden: DE dorsales externas, DI dorsales internas, VE ventrales externas y VI ventrales internas. Tibia posterior: número de espinas apicales internas en el ápice de las tibias derecha e izquierda.

Table 4.- Leg spurs in Pterolepis cordubensis. Fore tibia: number of spurs in the following order: DE upper outer margin, DI upper iner margin, VE outer lower margin and VI inner lower margin. Hind tibia: number of mid spurs in the tip of right and left legs.

\begin{tabular}{lccccccc}
\hline & \multicolumn{7}{c}{ Pterolepis cordubensis } \\
\hline Localidad & sexo & DE & DI & VE & VI & Tibia posterior \\
& & 4 & $2 / 0$ & 6 & 6 & derecha-izquierda \\
\hline Córdoba, Holotipo. MNCN & macho & & 4 & & & & \\
\hline
\end{tabular}

(CH2173, Alicante, Sierra de Menachaor bei Ibi) con el que estableció la sinonimia de esta especie con P. spoliata (Heller, 1988), damos por válida la especie Pterolepis grallata (Pantel, 1886).

En la Tabla 6 se recogen los caracteres que identifican a esta especie: la morfología del cerco masculino con un diente basal y $10^{\circ}$ terguito abdominal del macho sin estilos (Fig. 1A); titiladores con la porción proximal expandida y perpendicular a la porción distal que es recta, alargada y con el ápice redondeado (Fig. 1B); y placa subgenital de la hembra trapezoidal, ancha y con la escotadura distal amplia y poco profunda (Fig. 1G). Todas estas estructuras son completamente diferentes a las que presenta $P$. spoliata con la que se estableció la sinonimia (Figs. 1C, 1D y $1 \mathrm{H}$, respectivamente para cada una de ellas). El ejemplar de H. G. Heller pertenece a $P$. spoliata y no a $P$. grallata. Probable- mente la confusión se deriva de que ese ejemplar carece de espinas en el ápice de la tibia posterior, por lo que Heller pudo haberlo atribuido a Rhacocleis, pero al no observar diferencias con $P$. spoliata, (ya que en realidad se trata de esta misma especie), estableció la sinonimia entre ambas. Puesto que además, como el propio autor indica, el canto de este ejemplar concuerda en el canto con $P$. spoliata (Heller, 1988).

La descripción de Pantel (1886) es bastante completa e incluso se aportan dibujos del cerco del macho y la placa subgenital de la hembra. Si bien, como era habitual en esa época, no se alude a los titiladores. Harz (1969) ilustra esta estructura así como el $10^{\circ}$ terguito del macho. La forma de los titiladores de esta especie es muy particular y difiere del patrón que en general presentan el resto de especies de Pterolepis ibéricas, pues es la única que

Tabla 5.- Ejemplares empleados en los análisis filogenéticos.

Table 5.- Specimens used in phylogenetic analyses.

\begin{tabular}{llllll}
\hline Ind. & Especie & Sexo & Fecha & Localidad & Colector \\
\hline I & P. spoliata & Hembra & $29 / \mathrm{VII}$ & Granada: Almegijar & E. Recuero \& M. García París \\
II & P. spoliata & Hembra & $29 / \mathrm{VII}$ & Granada: Almegijar & E. Recuero \& M. García París \\
III & P. spoliata & Hembra & $29 / \mathrm{VII}$ & Granada: Almegijar & E. Recuero \& M. García París \\
IV & P. spoliata & Hembra & $20 / \mathrm{VI}$ & Sevilla: Cazalla de la Sierra & J. Gutiérrez Rodríguez \\
V & P. spoliata & Hembra & $20 / \mathrm{VI}$ & Sevilla: Cazalla de la Sierra & J. Gutiérrez Rodriguez \\
VI & P. grallata & Hembra & $01 / \mathrm{VII}$ & Madrid: Valdelaguna & J. Gutiérrez Rodríguez \\
VII & P. grallata & Hembra & $08 / \mathrm{VII}$ & Madrid: Valdelaguna & J. Gutiérrez Rodríguez \\
VIII & P. grallata & Macho & $15 / \mathrm{VII}$ & Madrid: Valdelaguna & J. Gutiérrez Rodríguez \\
IX & T. tessellata & Macho & $06 / \mathrm{VIII}$ & Madrid: Colmenar Viejo & J. Gutiérrez Rodriguez \\
\hline
\end{tabular}


presenta la porción proximal aplanada de cuyo centro surge perpendicularmente la porción distal en forma de espátula.

Los 33 ejemplares estudiados de $P$. grallata poseen el borde dorsal interno de las tibias anteriores sin espinas por lo que también, según el criterio de Willemse \& Willemse (2005), se encuadran en el género Pterolepis.

\section{Pterolepis spoliata Rambur, 1838}

Pterolepis spoliata Rambur, 1838: 60 - Caudell, 1908: 12 Harz, 1969: 440 - Otte, 1997: 295 - Heller et al., 1998: 38

- Willemse \& Willemse, 2005: 268

Pterolepis spoliata var. minor Bolívar, 1900: 14

Pterolepis minor Bolívar: Galvagni, 1981: 174

Pterolepis nevadensis Galvagni, 1981: 181

Pterolepis nadigi Galvagni, 1981: 188

Pterolepis raggei Galvagni, 1981: 194

Pterolepis llorenteae Galvagni, 1981: 205

Pterolepis pascuali Galvagni, 1981: 209

Pterolepis kaltenbachi Galvagni, 1981: 214

Pterolepis spoliata spoliata Rambur: Heller, 1988: 143

Pterolepis spoliata grallata (Pantel): Heller, 1988: 143

Pterolepis spoliata nevadensis Galvagni: Heller, 1988: 143

Pterolepis spoliata nadigi Galvagni: Heller, 1988: 143

Pterolepis spoliata pascuali Galvagni: Heller, 1988: 143

Pterolepis spoliata kaltenbachi Galvagni: Heller, 1988: 143

Pterolepis spoliata llorenteae Galvagni: Heller, 1988: 143

Pterolepis spoliata raggei Galvagni: Heller, 1988: 143

Es la especie ibérica del género con distribución más amplia, extendiéndose por la mitad meridional de la península. Bolívar (1900) la cita desde Cádiz a Cartagena, si bien consideró como variedad a los ejemplares de la provincia más occidental. El trabajo de Galvagni (1981) supuso la elevación a rango de especie de la variedad, pasando a $P$. minor Bolívar, 1900 y la descripción de seis nuevas especies a partir, en parte, de ejemplares determinados con anterioridad como P. spoliata (Bolívar, 1900; Llorente, 1980; Pascual, 1978a, b). Así, describió P. nadigi, P. pascuali y $P$. nevadensis (en parte) y P. llorenteae. Estas especies presentaban pequeñas variaciones morfológicas, con lo que cada una de las poblaciones geográficas conocidas fue descrita como nueva especie. La separación morfológica de cada una de ellas es muy sutil en el caso de los machos y muy difícil o imposible en el caso de las hembras. Galvagni señala variaciones en la forma de los titiladores para $P$. minor o $P$. nadigi, $\tan$ distantes como las que le permiten la diferenciación de otras especies. Por lo tanto en unos casos esas diferencias sirven para separar especies diferentes y en otros no. De igual modo, la forma de la concavidad o escotadura del borde posterior de la

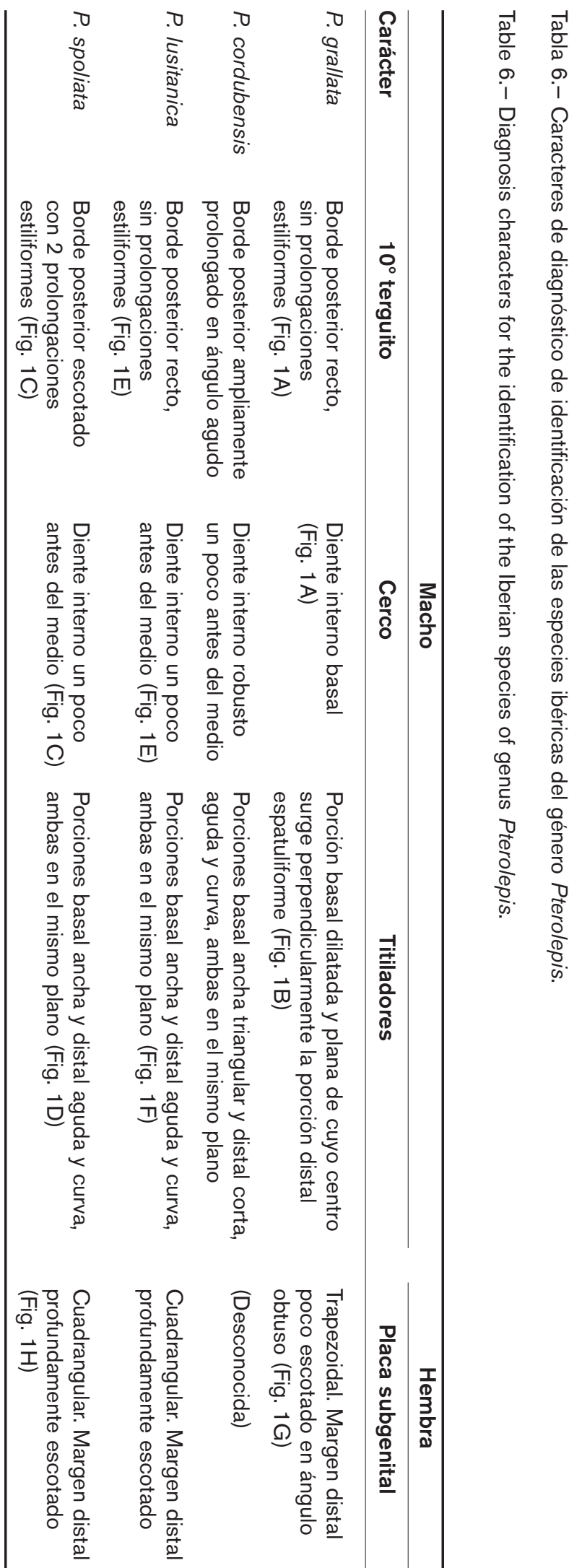

Graellsia, 66(2), Diciembre 2010, pp. 131-148 - ISSN: 0367-5041 doi:10.3989/graellsia.2010.v66.008 


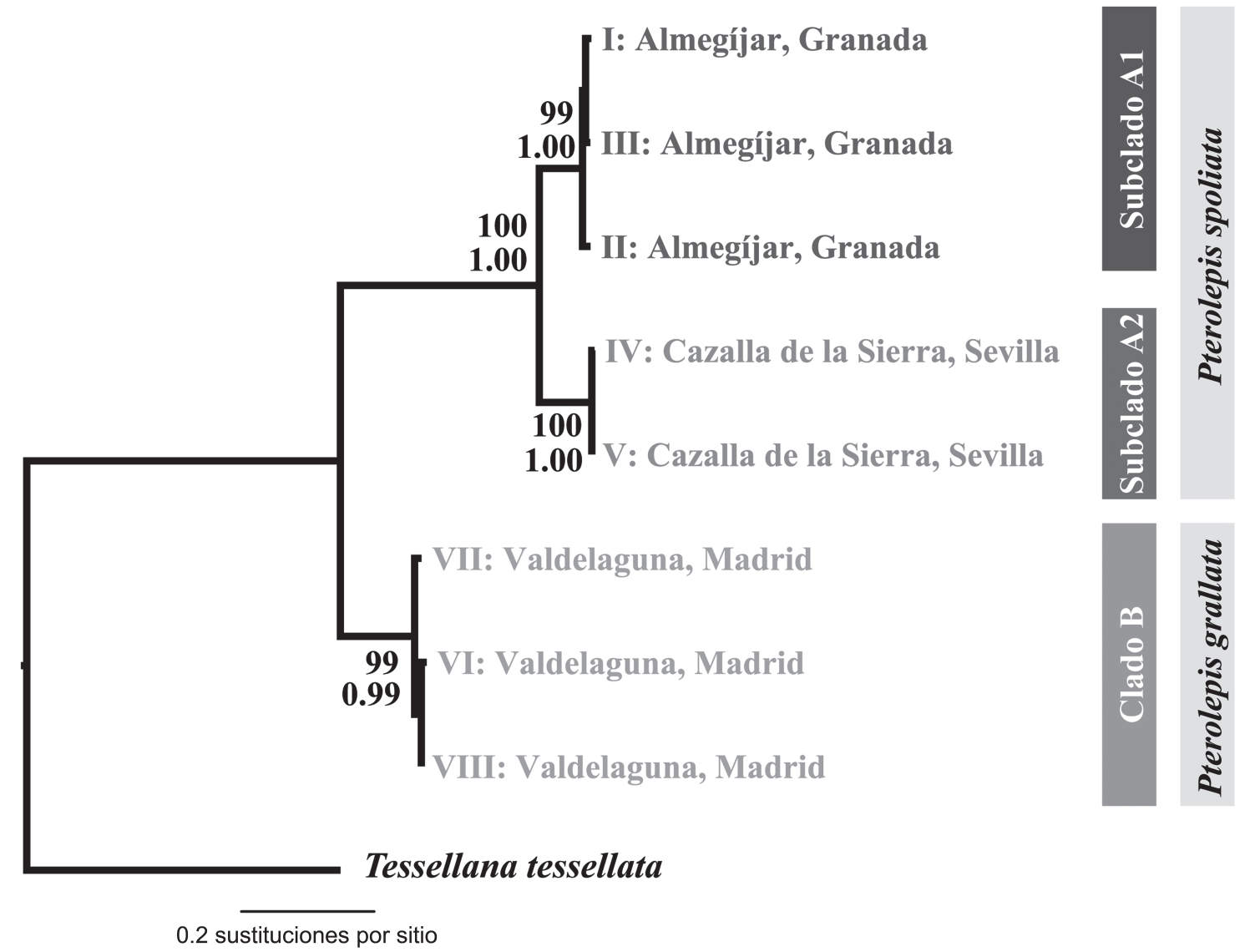

Fig. 2.- Hipótesis filogenética basada en los análisis de máxima verosimilitud. Los números superiores indican los valores de bootstrap para el análisis ML. Los valores inferiores indican la probabilidad posterior estimada para cada nodo en el análisis bayesiano.

Fig. 2.- Phylogenetic hypothesis based on maximum likelihood analysis. Numbers next to branches indicate bootstrap values associated with maximum likelihood analysis (up) and Bayesian analysis posterior probabilities (down).

placa subgenital femenina y la curvatura del cerco masculino, puede presentarse más o menos redondeados o cuadrangulares dentro de individuos de la misma zona geográfica, con lo que aparecen formas intermedias y de difícil asignación a una especie en concreto. Heller (1988) señala que no aprecia diferencias morfológicas ni tampoco se aprecian variaciones en el canto, por lo que este autor relega las especies de Galvagni al rango de subespecies.

El estudio de 74 ejemplares de esta especie, entre los que figuran algunos de los tipos de Galvagni, nos posiciona en la postura de Heller de considerarlos a todos por el momento como pertenecientes a $P$. spoliata. El estudio de ejemplares procedentes de nuevas localidades evidencia diferencias morfológicas intermedias o próximas a los taxones de
Galvagni, como en el caso del material de las provincias de Córdoba o Madrid. No obstante el análisis filogenético evidencia distancias genéticas entre las poblaciones que podría indicar la validez de las subespecies como especies válidas. Para confirmar este punto, es necesario la captura de material nuevo y su preservación en alcohol absoluto de las diferentes localidades para su secuenciación.

\section{Pterolepis lusitanica (Bolívar, 1900)}

Scirtobaenus lusitanicus Bolívar, 1900: 15 - Caudell, 1908: 5 Rhacocleis lusitanica (Bolívar): Harz, 1969: 440 - Otte, 1997: 295 - Willemse \& Willemse, 2005: 265

Pterolepis lusitanica (Bolívar): Heller, 1988: 143 - Heller et al., 1998: 38 
Tabla 7.- Divergencias genéticas medias y valor mínimo y máximo (basadas en distancias sin corregir) entre los ejemplares del estudio filogenético.

Table 7.- Average, minimum and maximum genetic divergence (uncorrected distances) among samples.

\begin{tabular}{lccc}
\hline & P. spoliata (Granada) & P. spoliata (Sevilla) & P. grallata \\
\hline P. spoliata (Granada) & $0,760 \%$ & & \\
P. spoliata (Sevilla) & $(0,608-0,912 \%)$ & & \\
P. grallata & $5,294 \%$ & $0,152 \%$ & \\
& $(5,015-5,471 \%)$ & & $0,709 \%$ \\
T. tessellata & $12,817 \%$ & $(12,158-12,76 \%)$ & $(0,304-1,064 \%)$ \\
& $(12,462-13,222 \%)$ & $20,137 \%$ & $18,541 \%$ \\
& $19,858 \%$ & $(20,061-20,213 \%)$ & $(18,389-18,693 \%)$ \\
\hline
\end{tabular}

Bolívar (1900) describe esta especie, a partir de un único ejemplar macho, del siguiente modo: "á $S$. grallato tantum differt: Statura majore, femoribus anticis subtus nigro trispinosis, tibiis anticis supra intus unispinosis; segmento anali ? medio lobo postice rectangulari, medio longitudinaliter sulcato. Cercis valde curvatis acutis". [Se diferencia $S$. grallato en: tamaño mayor. Fémures anteriores con tres espinas ventrales negras. Tibias anteriores con el margen interno superior uniespinoso. Segmento anal del macho con un lóbulo medio rectangular surcado en el centro. Cerco muy curvado y agudo]. Se omite en la descripción que el cerco del macho posee diente interno.

Curiosamente el lectotipo es el único ejemplar estudiado de esta especie con espinulación irregular en las tibias anteriores (ver Tabla 3). Además como se indica en la tabla esta espina sólo aparece en la tibia anterior izquierda que también es irregular en la espinación ventral externa (5/6 respectivamente). El borde superior interno de la tibia anterior derecha es liso como en los otros 9 ejemplares estudiados. Las espinas ventrales del fémur anterior (aunque en la propia clave de Bolívar (1990) este carácter está equivocado, pues indica fémur posterior) varían de 2 a 4, incluso para el mismo ejemplar entre las patas izquierda y derecha en los ejemplares estudiados. Son muy características la forma del décimo terguito y el cerco del macho (Fig. 1E) que es agudo y curvado como el de $P$. spoliata pero más robusto y angular, recuerda al de $P$. cordubensis. Los titiladores presentan la porción proximal más estrecha que $P$. spoliata y con la porción distal curvada y aguda desde el último tercio (Fig. 1F).
Los 10 ejemplares estudiados de esta especie (salvo la pata anterior izquierda del lectotipo) tienen las tibias anteriores sin espinas dorsales internas, por lo que de acuerdo con el criterio de Willemse \& Willemse (2005) se encuadran dentro del género Pterolepis.

\section{Pterolepis cordubensis Bolívar, 1900}

Pterolepis cordubensis Bolívar, 1900: 14 - Caudell, 1908: 12 Harz, 1969: 426 - Otte, 1997: 293 - Heller et al., 1998: 37

- Willemse \& Willemse, 2005: 268

Se ha estudiado el único ejemplar conocido de esta especie aportándose los datos correspondientes a la espinulación tibial (Tabla 4). Lamentablemente el titilador de esta especie no se conserva, se ha perdido parcialmente, quedando únicamente el titilador izquierdo mutilado adherido a una etiqueta junto con el ejemplar. De este fragmento triangular, junto con la figura de Harz (1969) se deduce que la forma del mismo es acorde con las especies ibéricas $P$. spoliata y $P$. lusitanica, con una porción proximal ancha que se adelgaza hacia la porción distal delgada, aguda y arqueada en forma de gancho. Este ejemplar es bastante robusto y recuerda a las especies norteafricanas, si bien como se ha indicado el cerco del macho le asemeja con $P$. lusitanica.

\section{Conclusiones}

El estudio morfológico del material ibérico (118 ejemplares) ha puesto de manifiesto la invalidez del 
carácter de la presencia o ausencia de dos espinas centrales del ápice de la tibia posterior por su variación individual, intraespecífica e intrapoblacional (Tablas 1-3). De acuerdo con el criterio de Heller et al. (1998) y con el propuesto por Willemse \& Willemse (2005), se descarta el carácter utilizado por Brunner von Wattenwyl (1893) para separar los géneros Rhacocleis y Pterolepis. Asímismo se constata la validez y constancia del carácter propuesto por estos autores en relación a las espinas del borde superior interno de las tibias anteriores. Este carácter posiciona a las especies ibéricas consideradas en el género Pterolepis, pues carecen de espinas en el margen dorsal interno de la tibia anterior (salvo dos ejemplares asimétricos).

Para el material estudiado no ibérico (45 ejemplares) también se confirma la variabilidad intraespecífica, intrapoblacional e individual de la espinación del ápice de la tibia posterior.

Damos por válida la especie Pterolepis grallata (Pantel, 1886) puesto que sus caracteres morfológicos son completamente diferentes a P. spoliata y se evidencia en el análisis filogenético, con lo que se invalida la sinonimia establecida por Heller (1988).

\section{Agradecimientos}

Queremos expresar nuestro agradecimiento a las personas que gestionan la colección de Entomología del MNCN de Madrid: Dr. Miguel Ángel Alonso Zarazaga, Mercedes París García, Mercedes Hitado y Marisa Peláez por su disposición y colaboración. También a las entidades que sufragan este estudio: Secretaría de Estado de Investigación mediante la concesión de los Proyectos CGL2007-66786C08-06 y CGL2007-64621 del MCI y Plan Propio de la Universidad de Almería por la concesión de una Ayuda (EST2008/001) para la realización de la estancia en el MNCN de Madrid.

A los doctores Raimundo Outerelo de la Universidad Complutense de Madrid, Felipe Pascual de Universidad de Granada y Juan José Presa de la Universidad de Murcia por prestarnos material de estudio. A Manuel Baena por la cesión de parte del material estudiado. A Sonia Ferreira del CIBIO-UP de Portugal por el préstamo de material portugués. Al Dr. Arturo Baz del Departamento de Zoología y Antropología Física de la Universidad de Alcalá de Henares por confiarnos el material ortopterológico capturado mediante trampas de carroña. Y al Dr. K.-G. Heller por prestarnos para su estudio 6 ejemplares de Pterolepis ibéricos.

Y muy especialmente al Dr. Antonio Aguirre Segura por confiarnos su colección de material almeriense, discusión y revisión del manuscrito.

A Ernesto Recuero y Mario García París por las muestras aportadas al estudio. A Patricia Cabezas y Federico Abascal por la ayuda en la realización de los análisis filogenéticos.

\section{Referencias}

Bolívar, I., 1900. Catálogo sinóptico de los ortópteros de la fauna ibérica. Annaes de Sciências Naturaes, [1899], 6: 1-28.

Brunner von Wattenwyl, C., 1893. Révision du systéme des Orthopteres et description des espécesrapportées par M. Leonardo Fea de Birmanie. Annali del Museo Civico di Storia naturale di Genova "Giacomo Doria", Serie 2, 13(33): 1-230, 6 pl.

Caudell, A. N., 1908. Orthoptera Fam. Locustidae. Subfam. Decticinae. Genera Insectorum, 72: 1-43, 2 pl.

Defaut, B., 2001. Actualisation Taxonomique et Nomenclaturale du "Synopsis des Orthopters de France". Matériaux Entomocénotiques, 6: 107-112.

Eades, D. C. \& Otte, D., Orthoptera Species File Online. Versión 2.0/3.5. <http://Orthoptera.SpeciesFile.org>. Consultada 14/03/2009.

Folmer, O., Black, M., Hoeh, W., Lutz, R. \& Vrijenhoek, R., 1994. DNA primers for amplification of mitochondrial cytochrome $c$ oxidase subunit I from diverse metazoan invertebrates. Molecular Marine Biology and Biotechnology, 3: 294-299.

Galvagni, A., 1981. Studio revisionale sulle specie iberiche del genere Pterolepis Rambur, 1838 (Insecta, Ensifera, Tettigoniidae, Decticinae). Atti dell'Accademia Roveretana degli Agiati, Serie VI, [1980], 20: 169-232.

Galvagni, A., 1984. Apporto alla conoscenza del genere Rhacocleis Fieber, 1853, in Algeria, con descrizione di R. silviarum n. sp. (Insecta, Ensifera, Tettigoniidae, Decticinae). Atti dell'Accademia Roveretana degli Agiati, Serie VI, 23: 85-102.

Galvagni, A., 1985. Il genere Pterolepis Rambur, 1838 nelle sue specie dell'Africa Minore e della Sicilia (Insecta, Ensifera, Tettigoniidae, Decticinae). Atti dell'Accademia Roveretana degli Agiati, Serie VI, [1984], 24: 135-158.

Guindon, S. \& Gascuel, O., 2003. A simple, fast, and accurate algorithm to estimate large phylogenies by maximum likelihood. Systematic Biology, 52(5): 696-704.

Harz, K., 1969. Die Orthopteren Europas I. Series Entomologica, 5: 1-749.

Heller, K.-G., 1988. Bioakustik der europäischen Laubheusschrecken. Ökologie in Forschung und Anwendung, 1: 1-358.

Heller, K.-G., Korsunouvskaya, O., Ragge, D. R., Vedenina, V., Willemse, F., Zhantiev, R. D. \& Frantsevich, L., 1998. Check-list of European Orthoptera. Articulata, 7: 1-61.

Llorente, V., 1980. Los ortopteroides del Coto de Doñana (Huelva). Eos, 54: 117-165.

Machordom, A., Araujo, R., Erpenbeck, D. \& Ramos, M-A., 2003. Phylogeography and conservation genetics of endangered European Margaritiferidae 
(Bivalvia: Unionoidea). Biological Journal of the Linnean Society, 78: 235-252.

Otte, D., 1997. Tettigonioidea. Orthoptera Species File, 7: 1-373.

Pantel, P. J., 1886. Contribution à l'orthoptérologie de l'Espagne Centrale. Anales de la Sociedad Española de Historia Natural, XV: 237-287

Pascual, F., 1978a. Estudio preliminar de los ortópteros de Sierra Nevada. III: Distribución ecológica. Trabajos y Monografías del Departamento de Zoología - Universidad de Granada, (N.S.), 1(2): 65121.

Pascual, F., 1978b. Estudio preliminar de los ortópteros de Sierra Nevada. IV: Distribución altitudinal. Boletín de la Asociación española de Entomología, 2: 49-63.

Ponel, P., Asmodé, J.-F., Gadum, S., Joyeux, S. \& Orsini, P., 1998. Nouvelles doneés sur la répatition geographique, l'écologie et la phénologie de Rhacocleis poneli Harz \& Voisin, 1987 (Orthoptera, Decticinae). Bulletin de la Société entomologique de France, 103: 341-347.

Posada, D. \& Crandall, K. A., 1998. Modeltest: testing the model of DNA substitution. Bioinformatics, 14: 817-818.

Rambaut, A. \& Drummond, A. J., 2007. Tracer 1.4. http://beast.bio.ed.ac.uk/Tracer. (Acceso: 3 de junio de 2010).

Rambur, J. P., 1838. Faune entomologique de l'Andalousie, vol. 2(3). Bertrand. Paris. pp. 17-96.

Ronquist, F. \& Huelsenbeck, J. P., 2003. MrBayes 3: Bayesian phylogenetic inference under mixed models. Bioinformatics, 19: 1572-1574.

Swofford, D. L., 2002. PAUP*. Phylogenetic Analysis Using Parsimony (* and Other Methods), Version 4. Sinauer Associates. Sunderland, Massachusetts.
Uvarov, B. P., 1934. Studies in the Orthoptera of Turquey, Iraq and Syria. Eos, 10: 21-119.

Uvarov, B. P., 1942. New and less known southern Palaearctic Orthoptera. Transactions of the American Entomological Society, 67: 303-361.

Willemse, F. \& Willemse, L., 2005. Rhacoclesis Fieber 1853 and Pterolepis Rambur 1838, distinc species groups or not. Journal of Orthoptera Research, 14(2): 261-269.

Willemse, F. \& Willemse, L., 2008. An annotated checklist of the Orthoptera-Saltatoria from Greece including an updated bibliography. Articulata Beiheft, 13: $1-91$.

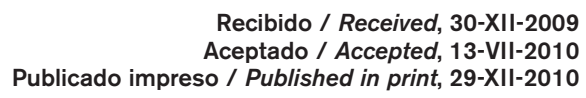




\section{Apéndice}

\section{Material examinado de Pterolepis grallata (Pantel, 1886):}

- 1 macho. COTIPO (roja impresa); Uclés (manuscrita a pluma); $S$. grallatus P. (manuscrita a pluma de I. Bolívar); MNCN Cat. Tipos $\mathrm{N}^{\circ} 7242$ (roja impresa). Pterolepis grallata (Pantel, 1886) P. Barranco det. 2009 (impresa). [MNCN de Madrid].

- 1 hembra. COTIPO (roja impresa); Uclés (manuscrita a pluma); Rhacocleis grallata (Pant.) V. Llorente det. 1992.; [MNCN Cat. Tipos No 7242 (roja impresa). Pterolepis grallata (Pantel, 1886) P. Barranco det. 2009 (impresa). [MNCN de Madrid].

- 1 macho. Cast. Branc. P. Bonet (manuscrita a pluma); Scirtobaenus Pant. grallatus Pantel (manuscrita a pluma de I. Bolívar). Pterolepis grallata (Pantel, 1886) P. Barranco det. 2009 (impresa). [MNCN de Madrid].

- 1 macho. Cast. Branc. P. Bonet (manuscrita a pluma); Rhacocleis grallata (Pant.) V. Llorente det. 1992 (manuscrita a tinta e impresa). Pterolepis grallata (Pantel, 1886) P. Barranco det. 2009 (impresa). [MNCN de Madrid].

- 1 macho. Jándula. Prov. de Jaén. X-1939. F. Escalera (impresa); Rhacocleis grallata (Pant.) V. Llorente det. 1992 (manuscrita a tinta e impresa). Pterolepis grallata (Pantel, 1886) P. Barranco det. 2009 (impresa). [MNCN de Madrid].

- 1 hembra. Jándula (Jaén). Gil Collado (manuscrita a pluma) Rhacocleis grallata (Pant.) V. Llorente det. 1992 (manuscrita a tinta e impresa). Pterolepis grallata (Pantel, 1886) P. Barranco det. 2009 (impresa). Pterolepis grallata (Pantel, 1886) P. Barranco del. 2009 (impresa). [MNCN de Madrid].

- 1 hembra. Talavera. P. Capelle (manuscrita a pluma). Pterolepis grallata (Pantel, 1886) P. Barranco det. 2009 (impresa). [MNCN de Madrid].

- 2 machos. Brunete. VII. 938 (manuscrita a pluma E. Morales); Prov. de Madrid. E. Morales (impresa); Rhacocleis grallata (Pant.) V. Llorente det. 1992 (manuscrita a tinta e impresa). Pterolepis grallata (Pantel, 1886) P. Barranco det. 2009 (impresa). [MNCN de Madrid].

- 1 hembra. Córdoba. Cascollán (manuscrita a pluma); Rhacocleis grallata (Pant.) V. Llorente det. 1992 (manuscrita a tinta e impresa). Pterolepis grallata (Pantel, 1886) P. Barranco det. 2009 (impresa). [MNCN de Madrid].

- 1 macho. El Pardo (El Goloso). Trampa Malaise. (9-15)-VIII-91. Nieves \& Rey leg. (impresa); Rhacocleis grallata (Pant.) V. Llorente det. 1993 (manuscrita a tinta e impresa). Pterolepis grallata (Pantel, 1886) P. Barranco det. 2009 (impresa). [MNCN de Madrid].

- 1 hembra. Ponte do Sabor. (I-5) 400 m. 15/9/00. 251/00. M.J. Miranda leg. 2000 (manuscrita a tinta e impresa); Rhacocleis grallata (Pant.) M.J. Miranda det. 2001 (manuscrita a tinta e impresa). Pterolepis grallata (Pantel, 1886) P. Barranco det. 2009 (impresa). [PBV].

- 1 macho. Km 5 Ctra. S. Calixto. Córdoba. España. F. Ferreras. 11-883. Dpto. Zool. Univ. Córdoba (manuscrita a tinta). Rhacocleis grallata (Pantel, 1886) P. Barranco det. 1994 (manuscrita a tinta e impresa). Pterolepis grallata (Pantel, 1886) P. Barranco det. 2009 (impresa). [PBV].

- 1 macho. Tiesas Quintanal. 1-8-1992. Santisteban del Puerto. Sa Morena. Jaén. España. J.C. Rodríguez leg. (impresa); Rhacocleis grallata (Pantel, 1886) P. Barranco det. 1992 (manuscrita a tinta e impresa). Pterolepis grallata (Pantel, 1886) P. Barranco det. 2009 (impresa). [PBV].

- 1 macho. El Porrosillo. Los Socabones. Jaén. 4-VII-2007. J. Maestre leg. (trampa de vinagre), (manuscrita a tinta). Rhacocleis grallata (Pantel, 1886). Pterolepis grallata (Pantel, 1886) P. Barranco det. 2009 (impresa). [PBV].

- 1 macho. El Porrosillo. Los Socabones. Jaén. 9-VII-2007. J. Maestre leg. (manuscrita a tinta). Rhacocleis grallata (Pantel, 1886) P. Barranco det. 2007 (manuscrita a tinta e impresa). Pterolepis grallata (Pantel, 1886) P. Barranco det. 2009 (impresa). [PBV].

- 1 macho ninfa, 1 hembra ninfa. Orusco. (480996, 4460577). $730 \mathrm{~m}$. Madrid. Junio 2006. Pradera, trampa de carroña con calamar. A. Baz leg. Pterolepis grallata (Pantel, 1886) P. Barranco det. 2009 (impresa). [UAH]

- 1 hembra. Orusco. (480996, 4460577). 730 m. Madrid. Agosto 2006. Pradera, trampa de carroña con calamar. A. Baz leg. Pterolepis grallata (Pantel, 1886) P. Barranco det. 2009 (impresa). [UAH]

- 1 hembra. Orusco. (480996, 4460577). 730 m. Madrid. Septiembre 2006. Pradera, trampa de carroña con calamar. A. Baz leg. Pterolepis grallata (Pantel, 1886) P. Barranco det. 2009 (impresa). [UAH]

- 1 macho. San Martín de la Vega. (456182, 4453188). 620 m. Madrid. Agosto 2006. Pradera, trampa de carroña con calamar. A. Baz leg. Pterolepis grallata (Pantel, 1886) P. Barranco det. 2009 (impresa). [UAH]

- 3 machos. Villamanta. (404260, 4464245). 610 m. Madrid. Agosto 2006. Bosque, matorral, pradera, trampa de carroña con calamar. A. Baz leg. Pterolepis grallata (Pantel, 1886) P. Barranco det. 2009 (impresa). [UAH]

- 1 hembra. Villaviciosa de Odón. (419855, 4473962), 540 m Madrid. Agosto 2006. Matorral, trampa de carroña con calamar. A. Baz leg. Pterolepis grallata (Pantel, 1886) P. Barranco det. 2009 (impresa). [UAH]

- 1 hembra. Chapinería. (397145, 4465864), 515 m. Madrid. Julio 2006. Bosque, trampa de carroña con calamar. A. Baz leg. Pterolepis grallata (Pantel, 1886) P. Barranco det. 2009 (impresa). [UAH]

- 1 hembra. Chapinería. (397145, 4465864), 515 m. Madrid. Agosto 2006. Bosque, trampa de carroña con calamar. A. Baz leg. P. Barranco det. 2009 (impresa). [UAH]

- 1 hembra ninfa. Villa del Prado. (386567, 4459281), 660 m. Madrid. Junio 2006. Pradera, trampa de carroña con calamar. A. Baz leg. Pterolepis grallata (Pantel, 1886) P. Barranco det. 2009 (impresa). [UAH]

- 1 macho. Villa del Prado. (386567, 4459281), 660 m. Madrid. Agosto 2006. Pradera, trampa de carroña con calamar. A. Baz leg. Pterolepis grallata (Pantel, 1886) P. Barranco det. 2009 (impresa). [UAH]. Fotografía SEM, Figs. 1A, 1B.

- 1 hembra. Villa del Prado. (386567, 4459281), 660 m. Madrid. Julio 2006. Matorral, trampa de carroña con calamar. A. Baz leg. Pterolepis grallata (Pantel, 1886) P. Barranco det. 2009 (impresa). [UAH]. Fotografía SEM, Fig. 1G.

- 1 macho. Amieiro. Alijó. PF3471. 164 m. 23/07/2006. Portugal. S. Ferreira leg. Pterolepis grallata (Pantel, 1886) P. Barranco det. 2009 (impresa). [CIBIO-UP]

- 1 macho. Palão. Freixo de Espada à Cinta. 29TPF8355 $630 \mathrm{~m}$. 10/10/2001. Portugal. Pedro Cardoso et al. leg. Pterolepis grallata (Pantel, 1886) P. Barranco det. 2009 (impresa). [CIBIO-UP]

- 1 hembra. Monte Barata. Castelo Branco. PD4495. 247 m. 13/07/2007. Portugal. S. Ferreira \& P. Sousa leg. Pterolepis grallata (Pantel, 1886) P. Barranco det. 2009 (impresa). [CIBIO-UP]

\section{Material examinado de Pterolepis spoliata (Pantel, 1886):}

- 1 macho. Algeciras. Mz Escalera (impresa); Pterolepis spoliata Bol. var (manuscrito a pluma de I. Bolívar); Pterolepis minor Bolívar det. A. Galvagni, 1981 (manuscrita a tinta e impresa). [MNCN de Madrid].

- 1 macho. Los Montes (Málaga) Marvier (manuscrito a pluma); Plesioallotypus. Pterolepis spoliata Rambur Galvagni, 1981 (roja impresa). Pterolepis spoliata Rambur V. Llorente det. 1989 (manuscrita a tinta e impresa). [MNCN de Madrid].

- 1 macho. Chiclana. Cepero (manuscrito a pluma); Lectoparatipo (roja impresa); Pterolepis minor Bolívar Galvagni, 1981 (roja impresa); MNCN Cat. Tipos $\mathrm{N}^{\mathrm{o}} 266$ (roja impresa). [MNCN de Madrid].

- 1 macho. Chiclana. Cepero (manuscrito a pluma); Pt. spoliata Rambur minor Bol. (manuscrita a pluma de I. Bolívar); Holotipo (impresa roja); Lectoholotypus Pterolepis minor Bolívar Galvagni, 1981 (roja impresa); MNCN Cat. Tipos No 267 (roja impresa). 
[MNCN de Madrid].

- 1 hembra. Chiclana. Cepero (manuscrito a pluma); Lectoallotypus Pterolepis minor Bolívar Galvagni, 1981 (roja impresa); MNCN Cat. Tipos No 268 (roja impresa). [MNCN de Madrid].

- 1 hembra. Chiclana. Cepero (manuscrito a pluma); Paralectotipo (roja impresa); Pt. spoliata Rambur minor Bol. (manuscrita a pluma de I. Bolívar); MNCN Cat. Tipos No 269 (roja impresa); [MNCN de Madrid].

- 2 hembras. Cartagena. Sanch. Gom. (manuscrito a pluma de I. Bolívar); [MNCN de Madrid].

- 1 macho. Cartagena. Sanch. Gom. (manuscrito a pluma de I. Bolívar); Pterolepis nadigi Galv. Galvagni, 1981 (manuscrita a tinta e impresa). Pterolepis nadigi Galvagni V. Llorente det. 1989 (manuscrita a tinta e impresa). [MNCN de Madrid].

- 1 macho. Palacio en la casa. Doñana (Huelva), 2-X-1968; A. Compte leg.; Holotypus Pterolepis llorenteae Galvagni, 1981 (roja impresa); Pterolepis llorenteae Galvagni V. Llorente det. 1989 (manuscrita a tinta e impresa); MNCN Cat. Tipos $\mathrm{N}^{\circ} 7320$ (roja impresa). [MNCN de Madrid].

- 1 macho. Pantel. P. spoliata Málaga (manuscrita a pluma de I. Bolívar); Pterolepis spoliata Rb (manuscrita a pluma de I. Bolívar); Pterolepis minor Bolívar? Galvagni, 1981 (manuscrita e impresa); [MNCN de Madrid].

- 1 hembra. Paterna. (manuscrita a pluma); [MNCN de Madrid].

- 1 hembra. Cehegín (Coto Real) Murcia, Mostacero (impresa); [MNCN de Madrid].

- 1 hembra. Sa de Gádor. Fondón. J. Martínez (impresa); [MNCN de Madrid].

- 1 hembra. Sierra Carbón. Algeciras. Escalera (manuscrita a pluma). [MNCN de Madrid].

- 1 hembra. Sin localidad; "difiere por tener espinas en los fémures intermedios. Loc??"; [MNCN de Madrid].

- 1 hembra. Granada. Cámara (manuscrita a pluma). [MNCN de Madrid].

- 1 macho. Jándula. Prov. de Jaén. XI-1932. F. Escalera (impresa); [MNCN de Madrid].

- 1 macho. Sa del Ayllo. Alcaudete. Jaén. 14-10-1990. M. Baena leg. (manuscrita); Pterolepis spoliata Rambur, 1838, P. Barranco det. 2009 (impresa). [PBV].

- 1 macho. Sa del Madroño. 30SWG7949. T.M. Albox. 800 m. Almería. 14-VII-1992. P. Barranco coll. (manuscrita e impresa); Pterolepis spoliata Rambur, 1838, P. Barranco det. 2009 (impresa). [PBV].

- 3 machos. Loma de la Capellanía. 30SWG8345. T.M. Taberno. 580 m. Almería. 14-VII-1992. P. Barranco coll. (manuscrita e impresa); Pterolepis spoliata Rambur, 1838, P. Barranco det. 2009 (impresa). [PBV].

- 1 hembra. Loma Mota. Sa de Gádor. 30SWG3586. T.M. Alhama. 900 m. Almería. 14-8-1992. P. Barranco leg. (manuscrita e impresa); Pterolepis spoliata Rambur, 1838, P. Barranco det. 2009 (impresa). [PBV].

- 1 hembra. Cerro Las Minas. Sa Cabrera. 30SWF8598. T.M. Turre. 550 m. Almería. 29-9-1992. P. Barranco leg. (manuscrita e impresa); Pterolepis spoliata Rambur, 1838, P. Barranco det. 2009 (impresa). [PBV].

- 1 hembra. Loma La Pata. Sa de Gádor. 30SWF3385. T.M. Enix. 1150 m. Almería. 3-9-1992. P. Barranco leg. (manuscrita e impresa); Pterolepis spoliata Rambur, 1838, P. Barranco det. 2009 (impresa). [PBV].

- 1 hembra. Los Cerrillos. 30SWG7811. T.M. Sorbas. Almería. 8-71993. P. Barranco leg. (manuscrita e impresa); Pterolepis spoliata Rambur, 1838, P. Barranco det. 2009 (impresa). [PBV].

- 1 hembra. Loma Mota. Sa de Gádor. 30SWG3586. T.M. Alhama. 900 m. Almería. 14-VIII-1992. P. Barranco leg. (manuscrita e impresa); Pterolepis spoliata Rambur, 1838, P. Barranco det. 2009 (impresa). [PBV].

- 1 hembra. Loma de las Colmenas. Sa de Gádor. UTM 30SWF1587, 1640 m. T.M. Fondón. Almería. 25-VII-2009. P. Barranco leg. (impresa); Pterolepis spoliata Rambur, 1838, P. Barranco det. 2009 (impresa). [PBV].

- 1 hembra. Gelibras. Turrillas. T.M. Almuñecar. Granada. 6-10-1996. J.A. de la Peña leg. (manuscrita); Pterolepis spoliata Rambur, 1838,
P. Barranco det. 2009 (impresa). [PBV].

- 1 hembra. Granada (manuscrita); Pterolepis spoliata Rambur, 1838, P. Barranco det. 2009 (impresa). Pterolepis spoliata Rambur, 1838, P. Barranco det. 2009. [PBV].

- 1 macho y 1 hembra. Los Villares. S $S^{\mathrm{a}}$ de Córdoba. Córdoba. 1-101991. J. Maldonado leg. (manuscrita); Pterolepis spoliata Rambur, 1838, P. Barranco det. 2009. [PBV].

- 1 macho. Antequera. Málaga. 9-1999. J.C. Rodríguez leg. (manuscrita); Pterolepis spoliata Rambur, 1838, P. Barranco det. 2009 (impresa). [PBV].

- 1 macho. Cementerio. Montoro. Córdoba. 30-8-2001. A.J. Márquez leg. (manuscrita); Pterolepis spoliata Rambur, 1838, P. Barranco det. 2009 (impresa). [PBV]

- 1 macho. Finca El Salado. St ${ }^{\mathrm{a}}$. $\mathrm{M}^{\mathrm{a}}$ de Trassierra. Córdoba. 6-9-2000. M.A. Morales- D. Mesa leg. (manuscrita); Pterolepis spoliata Rambur, 1838, P. Barranco det. 2009 (impresa). [PBV].

- 1 hembra. La Algaida. Entorno de Doñana. S. Lúcar de Barrameda. Cádiz. 16-VII-1997. P. Coello leg. (manuscrita); Pterolepis spoliata Rambur, 1838, P. Barranco det. 2009. [PBV].

- 1 hembra. Vega Andarax. Sa Nevada. T.M. Laujar. 1200 m. Almería 22-10-1988. P. Barranco leg. (manuscrita e impresa); Pterolepis spoliata Rambur, 1838, P. Barranco det. 2009. [PBV].

- 1 hembra. Vega Andarax. Sa Nevada. T.M. Laujar. 1000 m. Almería. 22-10-1988. P. Barranco leg. (manuscrita e impresa); Pterolepis spoliata Rambur, 1838, P. Barranco det. 2009 (impresa). [PBV]

- 1 hembra. Vega Andarax. Sa Nevada. T.M. Terque. Almería. 5-81989. P. Barranco leg. (manuscrita e impresa); Pterolepis spoliata Rambur, 1838, P. Barranco det. 2009 (impresa). [PBV].

- 1 hembra. Lancha de Cénes. Granada. 700 m. 3-XI-1977. F. Pascual leg. (manuscrita e impresa); Pterolepis nevadensis Galv.? det A. Galvagni 1981 (manuscrita e impresa); Pterolepis spoliata Rambur, 1838, P. Barranco det. 2009 (impresa). [UGR].

- 1 hembra. Huetor-Santillán. Granada. 2000 m. 23-7-1976. F. Pascual leg. (manuscrita e impresa); Pterolepis nevadensis Galv.? det A. Galvagni 1981 (manuscrita e impresa); Pterolepis spoliata Rambur, 1838, P. Barranco det. 2009 (impresa). [UGR]

- 1 macho. Valle del Río Lanjarón. Sierra Nevada. Granada. 1900 m. 30-7-1975. F. Pascual leg. (manuscrita e impresa); HOLOTYPUS Pterolepis pascuali Galvagni 1981 (roja impresa); Pterolepis spoliata var. minor Bolivar F. Pascual det. 1975 (manuscrita e impresa); Pterolepis spoliata Rambur, 1838, P. Barranco del. 2009 (impresa) [UGR].

- 1 hembra. Valle del Río Lanjarón. Sierra Nevada. Granada. 1900 m. 5-9-1975. F. Pascual leg. (manuscrita e impresa); ALLOTYPUS Pterolepis pascuali Galvagni 1981 (roja impresa); Pterolepis spoliata var. minor Bolivar F. Pascual det. 1975 (manuscrita e impresa); Pterolepis spoliata Rambur, 1838, P. Barranco det. 2009 (impresa). [UGR].

- 1 macho. P.N. Cabo Gata-Níjar. (Almería). Base del Cerro del Barronal. $36^{\circ} 44^{\prime} 01,1^{\prime \prime} \mathrm{N} / 02^{\circ} 08^{\prime} 29,2$ 'W. 6-8-2004. A. Aguirre \& D. Cañadas leg. (impresa); [AAS].

- 1 hembra. P.N. Cabo Gata-Níjar. (Almería). Base del Cerro del Barronal. 36 44'01,1'N /02 08'29,2”W. 30-9-2004. A. Aguirre \& D. Cañadas leg. (impresa); [AAS].

- 1 macho. P.N. Cabo Gata-Níjar. (Almería). Proximidades Torregarcía. $36^{\circ} 49^{\prime} 58,8^{\prime \prime} \mathrm{N} / 02^{\circ} 17^{\prime} 29,8^{\prime}$ W. 23-7-2004. A. Aguirre \& D. Cañadas leg. (impresa); [AAS]

- 1 hembra. P.N. Cabo Gata-Níjar. (Almería). Proximidades Torregarcía. $36^{\circ} 49^{\prime} 58,8^{\prime \prime} \mathrm{N} / 02^{\circ} 17^{\prime} 29,8^{\prime \prime} \mathrm{W}$. 10-9-2004. A. Aguirre $\&$ D. Cañadas leg. (impresa); [AAS].

- 1 macho. P.N. Cabo Gata-Níjar. (Almería). Albergue Las Amoladeras. $36^{\circ} 49^{\prime} 35,2^{\prime \prime} \mathrm{N} / 02^{\circ} 15,55,3^{\prime}$ 'W. 25-6-2004. A. Aguirre \& D. Cañadas leg. (impresa); [AAS]

- 1 macho. P.N. Cabo Gata-Níjar. (Almería). Albergue Las Amoladeras. $36^{\circ} 49^{\prime} 35,2^{\prime \prime} \mathrm{N} / 02^{\circ} 15^{\prime} 55,3^{\prime}$ 'W. 16-7-2004. A. Aguirre \& D. Cañadas leg. (impresa); [AAS].

- 1 macho. P.N. Cabo Gata-Níjar. (Almería). Albergue Las Amoladeras. $36^{\circ} 49^{\prime} 35,2{ }^{\prime \prime} \mathrm{N} / 02^{\circ} 15^{\prime} 55,3^{\prime}$ W. 17-9-2004. A. Aguirre \& D. Cañadas 
leg. (impresa); [AAS]

- 1 hembra. P.N. Cabo Gata-Níjar. (Almería). Albergue Las Amoladeras. $36^{\circ} 49^{\prime} 35,2^{\prime \prime} \mathrm{N} / 02^{\circ} 15^{\prime} 55,3^{\prime \prime} \mathrm{W}$. 24-9-2004. A. Aguirre \& D. Cañadas leg. (impresa); [AAS].

- 3 machos. P.N. Cabo Gata-Níjar. (Almería). Albergue Las Amoladeras. $36^{\circ} 49^{\prime} 35,2^{\prime \prime} \mathrm{N} / 02^{\circ} 15,55,3^{\prime \prime} \mathrm{W}$. 10-9-2004. A. Aguirre \& D. Cañadas leg. (impresa); [AAS].

- 1 hembra. P.N. Cabo Gata-Níjar. (Almería). Albergue Las Amoladeras. $36^{\circ} 49^{\prime} 35,2^{\prime \prime} \mathrm{N} / 02^{\circ} 15^{\prime} 55,3$ "W. 24-9-2004. A. Aguirre \& D. Cañadas leg. (impresa); [AAS].

- 1 macho. P.N. Cabo Gata-Níjar. (Almería). Obsevatorio de aves de las Salinas. $36^{\circ} 45^{\prime} 39,1^{\prime \prime} \mathrm{N} / 02^{\circ} 13$ ' $15,7^{\prime \prime}$ W. 30-7-2004. A. Aguirre \& D. Cañadas leg. (impresa); [AAS]. Fotografía SEM, Figs. 1C, 1D.

- 1 macho. P.N. Cabo Gata-Níjar. (Almería). Obsevatorio de aves de las Salinas. $36^{\circ} 45^{\prime} 39,1^{\prime \prime} \mathrm{N} / 02^{\circ} 13^{\prime} 15,7^{\prime \prime}$ W. 9-7-2004. A. Aguirre \& D. Cañadas leg. (impresa); [AAS].

- 1 hembra. P.N. C. Gata-Níjar. (Almería). Frente centro visitantes Las Amoladeras. 30-7-2004. A. Aguirre \& D. Cañadas leg. (impresa); [AAS].

- 1 hembra. P.N. Cabo Gata-Níjar. (Almería). Frente centro visitantes Las Amoladeras. 6-8-2004. A. Aguirre \& D. Cañadas leg. (impresa); [AAS]. Fotografía SEM, Fig. 1H.

- 1 macho. P.N. C. Gata-Níjar. (Almería). Frente centro visitantes Las Amoladeras. 3-9-2004. A. Aguirre \& D. Cañadas leg. (impresa); [AAS].

- 1 macho. La Hoya II. Águilas. (MU). 420 m. 30-5-1996. F. Hernández leg. (impresa); Pterolepis spoliata Rambur, 1838, P. Barranco det. 2009 (impresa). [UMU].

- 1 macho. C. Cuestas. Lorca. (MU). 660 m. 2-5-1996. F. Hernández leg. (impresa); Pterolepis spoliata Rambur, 1838, P. Barranco det. 2009 (impresa). [UMU].

- 1 macho. Casa del Toro. Lorca. (MU). 742 m. 2-5-1996. F. Hernández leg. (impresa); Pterolepis spoliata Rambur, 1838, P. Barranco det. 2009 (impresa). [UMU].

- 1 hembra. C. Torrecilla I. Lorca. (MU). 420 m. 20-7-1996. F. Hernández leg. (impresa); Pterolepis spoliata Rambur, 1838, P. Barranco det. 2009 (impresa). [UMU].

- 1 hembra. C. Torrecilla I. Lorca. (MU). 420 m. 20-8-1996. F. Hernández leg. (impresa); Pterolepis spoliata Rambur, 1838, P. Barranco det. 2009 (impresa). [UMU].

- 1 hembra. Colmenar Viejo. junio 1984. C. Sonyer (manuscrita); Pterolepis spoliata Rambur, 1838, P. Barranco det. 2009 (impresa). [UCM].

- 1 hembra. Aravaca. Madrid. 5-V-1988. P. Barrachina leg. (manuscrita); Pterolepis spoliata Rambur, 1838, P. Barranco det. 2009 (impresa). [UCM].

- 1 hembra. CH2171. [SPAIN: Malaga, Montes di Malaga, P. de Leon $\left(36^{\circ} 47^{\prime} \mathrm{N}, 4^{\circ} 23^{\prime} \mathrm{W}\right), 9$ viii 1984 , coll. Heller. Pterolepis spoliata spoliata Rambur, 1839]. Pterolepis spoliata Rambur, 1838, P. Barranco det. 2009 (impresa). [KGH].

- 1 macho. CH2173. [KGH]. [SPAIN. Alicante, Sierra de Menechaor bei Ibi (38³9’ N, 0³4’W), 1150 m, 15 ix 1980, coll. Heller. Pterolepis spoliata grallata (Pantel, 1886)]. Pterolepis spoliata
Rambur, 1838, P. Barranco det. 2009 (impresa).

- 1 macho y 1 hembra. CH2174 y CH2175. [SPAIN: Algeciras/Cádiz?, Sierra del Nino nw. Algeciras (36¹0’ N, 5³5’ W), 10-11 viii 1984, coll. Heller. Pterolepis spoliata ssp.]. Pterolepis spoliata Rambur, 1838, P. Barranco det. 2009 (impresa). [KGH].

- 1 macho y 1 hembra. CH2178 y CH2179. [SPAIN: Granada, Sierra Nevada bei Trevelez (3659’N, $3^{\circ} 16^{\prime}$ W), 9 viii 1984, coll. Heller. Pterolepis spoliata nevadensis Galvagni, 1981]. Pterolepis spoliata Rambur, 1838, P. Barranco det. 2009 (impresa). [KGH].

\section{Material examinado de Pterolepis lusitanica (Bolívar, 1900):}

- 1 macho. Cast. Branco. Portugal. P. Bonet (manuscrito a pluma); Scirtobaenus Pantel lusitanicus Bolivar (manuscrita a pluma de I. Bolívar). sine tillador (manuscrita a bolígrafo). Lectotipo (roja impresa). MNCN Cat. Tipos $\mathrm{N}^{\circ} 1311$ (roja impresa). Pterolepis lusitanica (Bolívar, 1900) P. Barranco det. 2009 (impresa). [MNCN de Madrid].

- 1 macho. Rhacocleis lusitanica V. Llorente det. 1992 (manuscrita a tinta e impresa). Pterolepis lusitanica (Bolívar, 1900) P. Barranco det. 2009 (impresa). [MNCN de Madrid].

- 1 hembra. Rhacocleis lusitanica V. Llorente det. 1992 (manuscrita a tinta e impresa). Pterolepis lusitanica (Bolívar, 1900) P. Barranco det. 2009 (impresa). [MNCN de Madrid].

- 1 macho. Monte Barata. Castelo Branco. PD4495. 247 m. 13/07/2007. Portugal. S. Ferreira \& P. Sousa leg. Pterolepis lusitanica (Bolívar, 1900) P. Barranco det. 2009 (impresa). [CIBIOUP]. Fotografía SEM, Figs. 1E, 1F.

- 1 hembra. Monte Barata. Castelo Branco. PD4495. 247 m. 13/07/2007. Portugal. S. Ferreira \& P. Sousa leg. Pterolepis lusitanica (Bolívar, 1900) P. Barranco det. 2009 (impresa). [CIBIOUP].

- 1 macho. Picota. Monchique. 29SNB4129. 770 m. 16/09/2007. Portugal. [CIBIO-UP]

- 2 machos. Rib. do Ruivo. Casas Novas da Cruz. Odemira. 29SNB3646. $100 \mathrm{~m}$. 03/09/2007. Portugal. Pterolepis lusitanica (Bolívar, 1900) P. Barranco det. 2009 (impresa). [CIBIO-UP]

- 1 hembra. Cruzes do Talurdo. Silves. 29SNB5328. $320 \mathrm{~m}$. 09/08/2007. Portugal. Pterolepis lusitanica (Bolívar, 1900) P. Barranco det. 2009 (impresa). [CIBIO-UP]

- 1 macho. Cruzes do Talurdo. Silves. 29SNB5328. 320 m. 05/09/2007. Portugal. Pterolepis lusitanica (Bolívar, 1900) P. Barranco det. 2009 (impresa). [CIBIO-UP]

\section{Material examinado de Pterolepis cordubensis Bolívar, 1900:}

-1 macho. Córdoba Coscollano (manuscrito a pluma); Pt. cordubensis Bol. (manuscrita a pluma de I. Bolívar). Holotipo (roja impresa). MNCN Cat. Tipos No 265 (roja impresa). [MNCN de Madrid]. 